\title{
The Design of the Integration Multifunction Terminal for Health Management Based on Android System
}

\author{
Ye Ding ${ }^{1, a}$, Shi Gang ${ }^{2, b}$ and Liu Bo ${ }^{3, c}$ \\ ${ }^{1,2,3}$ Shenyang Institute of Automation Chinese Academy of Sciences, China \\ ayeding@sia.cn, bsg0105@sia.cn, Cliubo@sia.cn
}

\begin{abstract}
Keywords: Health Information Collection Terminal; Android System; Intelligent Medical and Chronic Management.
\end{abstract}

\begin{abstract}
The paper introduces an Intelligent Health Information Management Terminal for grass-roots level medical department which is based on Android System to address the low degree of integration and intelligence of the current Health Collection Information Terminal. The new terminal adopts the integrating design of the structure and portable peripherals, which is convenient for doctor to carry. The abundant function of terminal including physiological and biochemical indicators detection, medical auxiliary diagnostic, remote export guide, electronic health document and health information management enhance the new terminal's intelligence degree. It could achieve that community doctors evaluate, screening and diagnose chronic for residents under the guidance of the expert doctors and help improve the level of basic medical service.
\end{abstract}

\section{Introduction}

With the rapid development of the ageing of population in our country, the number of patients with chronic diseases which is as the representative of hypertension and diabetes mellitus is increasing year by year and the younger trend of chronic diseases have shown as well. It is not only a problem of health, but also an important issue of public health which can bring danger to resident. However, the resource of medical apparatus is lack in the grass-roots level medical department and the general doctors' professional skill is low relatively. So they are lack of effective health management measures to take disease screening and auxiliary diagnostic for residents. Most of the patients go to the central hospital that causes the crowded phenomenon. The expert doctors have not enough time to guide and treat patient on chronic diseases. In addition, for the patients outside the hospital who has received treatment in the hospital, we are lack of a set of management mechanism to ward them effectively which leads to the complications.

There have been some integration medical devices of grass-roots level on the market in recent years. But these devices all have some deficiencies. One hand, they can only detect and collect physiological Indicator and can't give the intelligent evaluation and auxiliary diagnosis of the chronic for community doctors and residents. On the other hand, these devices don't have a built-in management information system and can't help doctor comprehensively understand patient's history health information. So they don't solve the grass-roots level medical demand.

For these demand at grass-roots level medical department and design deficiencies of the Health Information Terminal currently above-mentioned, we introduce a new design of Intelligent Health Information Management Terminal. The innovation of this new terminal is mainly reflected in the high level of integration and high intelligence. Firstly, the new terminal uses the Android operating system. It could provide the beautiful interface and the graphic operation. Secondly, the new terminal is not only collect physiological information and biochemical information, but also has a built-in intelligence expert medical model. It could assist the community doctor to take the health assessment and chronic disease screening for residents under the guidance of the remote expert doctors. Moreover, the new terminal has a built-in management information system. It could help build the electronic health records and manage the health information for residents. Last but not the least, the new terminal take low power and portable design and is convenient for community doctors to follow up the patients with chronic diseases outdoors. 


\section{Embedded Design of Terminal Based on Android}

Section Headings. The section headings are in boldface capital and lowercase letters. Second level headings are typed as part of the succeeding paragraph (like the subsection heading of this paragraph).

Embedded design of terminal based on Android is in Figure 1.

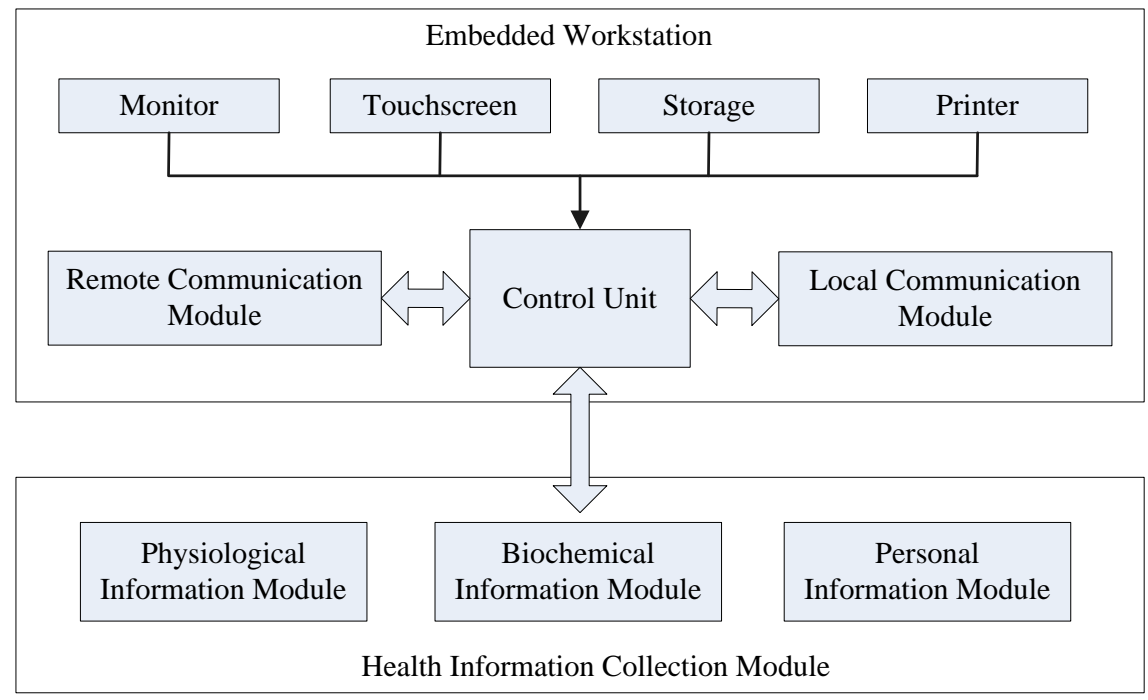

Figure 1. Embedded Design of Terminal Block Diagram

Embedded Workstation. Embedded Workstation is the core component of Intelligent Health Information Management Terminal. This component takes industrial motherboard and Android operating system as the control unit. It is not only guarantee the terminals low power consumption, but also provides the beautiful and smooth operator interface. The structure of Embedded Workstation is as follows:

- Human-Computer Interaction Unit: It's actually the tablet PC. Embedded Workstation can display the friendly application interface by monitor. Doctor could operate the equipment by touch screen.

- Remote Communication Module: This communication module is used to communicate to remote medical server. One hand it backs the health information up to the remote database, on the other hand it could take the remote expert guidance to local that assists medical decision and achieve the diagnosis online.

- Local Communication Module: This local module is used to communicate to health information collection module and collect personal information, physiological information and biochemical information to the terminal.

- Integrated Application Peripherals: Embedded Workstation integrates printer, RFID, Storage. It could achieve the function of identification, storage and printing on data and image.

Health Information Detection Module. This function module uses health information intelligent collection technology to achieve the physiological indicators and biochemical indicators rapid collection, while form the personal physical examination report. It can provide the reliable data sources to health assessment, chronic disease screening, auxiliary diagnostic and chronic disease management. The detailed physiological indicators and biochemical indicators as block 1 . 
Table 1. Health Information Detection Indicator

\begin{tabular}{|l|l|}
\hline Indicator Species & Details \\
\hline \multirow{5}{*}{ Physiological Indicator } & Electrocardiogram(ECG) \\
\cline { 2 - 2 } & Respiratory Rate \\
\cline { 2 - 2 } & Blood Oxygen \\
\cline { 2 - 2 } & Body Temperature \\
\cline { 2 - 2 } & Blood Pressure \\
\cline { 2 - 2 } Blood sugar Indicator & Pulse \\
\hline \multirow{5}{*}{ Gipid Indicator } & Clucose \\
\hline \multirow{5}{*}{ Routine Urine Indicator } & Triglycerides(TRIG) \\
\cline { 2 - 2 } & High-density Lipoprotein(HDL) \\
\cline { 2 - 2 } & Low-density Lipoprotein(LDL) \\
\hline & Leukocyte (LEU), \\
\cline { 2 - 2 } & Nitrite (NIT) \\
\cline { 2 - 2 } & Urobilinogen (UBG) \\
\cline { 2 - 2 } & Protein (PRO) \\
\cline { 2 - 2 } & PH \\
\cline { 2 - 2 } & Blood(BLD), \\
\cline { 2 - 2 } & Sitamin(VC) \\
\hline & Specific Gravity (SG) \\
\hline & Ketone Body (KET) \\
\hline & Bilirubin (BIL) \\
\cline { 2 - 2 } & Glucose(GLU) \\
\hline &
\end{tabular}

ECG monitoring is an important mean of diagnosing cardiovascular disease. The long record of dynamic ECG signal could improve the detection ability of ECG changes and reveal weak change on state of supine. It could also help acknowledge the relationship between these changes and Heart Rate, diary symptom, postural and so on.

Respiratory rate analysis algorithm is based on ECG electrical axis analysis method. The method takes the orthogonal ECG lead. According to calculate the square of the orthogonal ECG lead wave group, we can calculate relative change of electrical axis and export breathing curve. The export breathing curve and the real breathing curve have a high correlation coefficient. So the real Respiratory rate could be calculated.

The Blood Oxygen Measurement of Terminal is mainly based on the degree that hematoglobulin and reduced hemoglobin absorb different wavelengths of light. Oxygen probe uses the two specific wavelengths of light to go through the upper part of finger, while silicon photovoltaic cell receives transmitted light and generates an electrical signal. Then the result could be gotten by calculating the ratio of $\mathrm{AC}$ and $\mathrm{DC}$ components of the two lights.

The body temperature detection uses the Infrared detection technology. It is composed by an optical system, a photo detector, a signal amplifier and signal processing. Optical system convergences ear cavity and infrared radiation energy of tympanic. The size of the field of view is determined by the optical measurement system parts and their location. Infrared energy is focused on the photo detector and converts into a corresponding electrical signal. The signal passes through an amplifier and a signal processing circuit, in according with the algorithm and after the instrument within the target emissivity correction to digital signals, the master CPU unit converts the digital signal to a temperature value.

Non-invasive Blood Pressure measurement of Intelligent Collection Terminal mainly uses oscillography to achieve the precision measurement. Inflatable cuff is used to block arterial blood flow. During the process of slow blowing off, detection is from the vibration wave of vessel wall and 
finds out the intrinsic relationship between envelope of vibration wave and arterial pressure. Then the measurement result of Blood Pressure is gotten. In addition, patient status alarm and system status alarm should be added to monitor measurement state. As oscillometric measuring time could not be too long, we also design the function of blood pressure cuff quickly blowing off and Cuff overvoltage protecting to keep the patient safety during measurement.

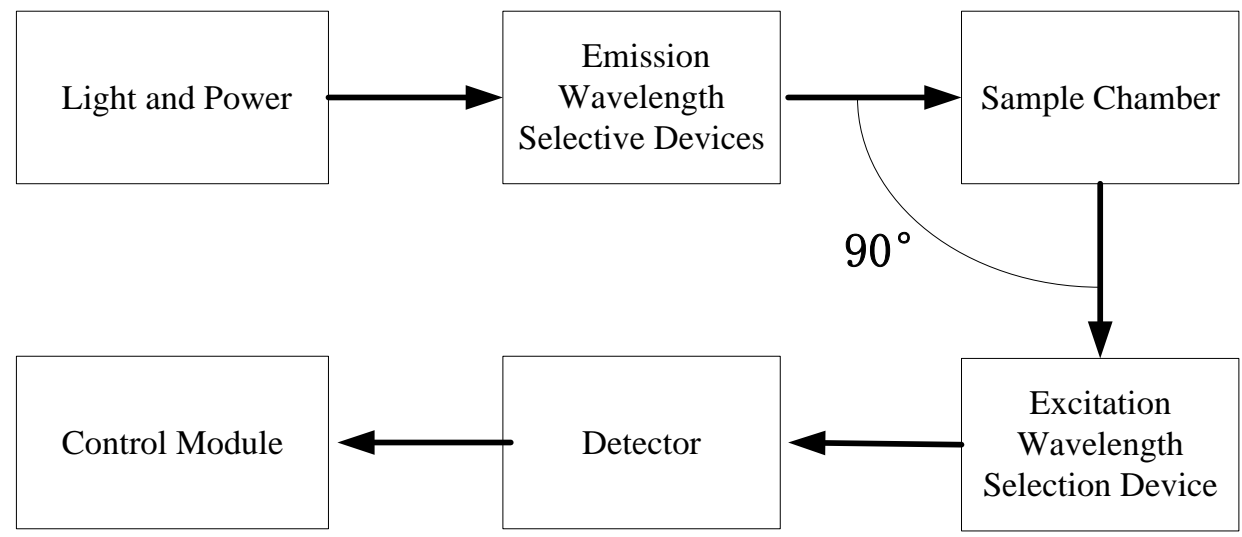

Figure 2. Dry Chemical Detection Diagram

The test strip takes the dry chemical method. The filter paper is the carrier for it and drying after every reagent component dipping as the reagent layer. Liquid immerse reagent strip and react with the reagent. Then the color will change. Lighter reflectance value is, the concentration of an ingredient in the liquid is higher.

\section{The Multifunction Design of Intelligent Terminal}

Medical Auxiliary Diagnostic. The terminal has a built-in intelligence expert medical model. It is shown in Figure.3.The import of the model will show Community doctors as questionnaire. For the resident who coming to the community for screening, the general doctor could get the resident's chronic risk factors, clinical symptoms and personal history by physical examination such as visual examination, interrogation, auscultation, palpation and so on, fill in the questionnaire at the same time. The model will assess, reasoning and analyze the content of the questionnaire and collected physiological indicators and biochemical indicators. Then it can obtain the evaluation results including risk assessment, Condition analysis, medication recommendation Lifestyle advices and transfer treatment advices. The results could assist community doctors to diagnose disease for residents.

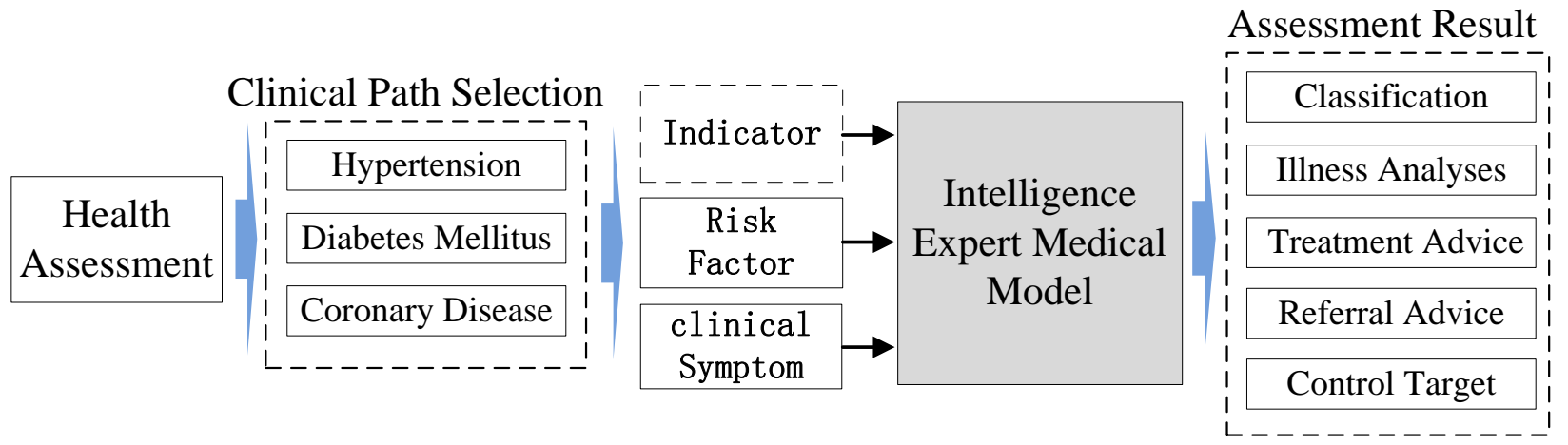

Figure 3. Intelligence Expert Medical Model Diagram

Remote Export Guide. If community doctors meet some incurable diseases which not be diagnosed during the health assessment and chronic disease screening. They could use the remote medical module send the local real-time health information to cloud intelligent expert medical system. According to this health information on patient the expert doctors who come from the central hospital lay down the individualized approach or give the transfer treatment advices in support of the cloud 
intelligence expert medical model. Then we achieve that the community doctors take the health assessment and chronic disease screening to the residents under the guidance of the expert doctors.

Electronic Health Document. According to the identity information collection, the community doctors build the personal health records for residents who come to be screening first. Terminal integrates RFID identity read module and can collect the information of resident's second-generation ID card rapidly. It is not only convenient for community doctors to build the health document for resident, but also ensure the personal information truth and effectiveness during the health assessment and chronic disease screening. Moreover, terminal could store every medical record of residents including physical examination report, transfer treatment record and so on.

Health Information Management. The terminal can provide informatization management of electronic medical case for that the community doctors could manage the patients with chronic diseases effectively. We take SQLite database technology to management the electronic record and ensure that the terminal can store the patients' previous screening and diagnosis report in the community including health assessment report, diagnosis and treatment report of chronic diseases and follow up report of chronic. The number of local medical case storage can be up to 5000. The search of health information management database supports multi condition query and fuzzy query and make sure that the operator could take and overlook the case rapidly. The database can also suppose the intelligent remote backup technology. It provides the backup, update and download from the remote server. In this way we achieve the efficient management and maintenance to the health information management database.

\section{The Integration Portable Design of Intelligent Terminal}

Considering the shortcoming of low integration about traditional collection terminal, we integrate the Embedded Workstation and Physiological Information Detection Module into a multi-function instrument. The electronic components on motherboard all take the patch style to reduce the space possibly. The instrument's shell adopts high strength synthetic plastics to ensure the light weight. The external features of the terminal is shown in Figure 4.

In terms of portable design, we communicate the physiological detection front-end (blood pressure cuff, oxygen finger clip, cochlear gun, ECG lead), biochemical detection device, second-generation ID card reader and printer to instrument by external way. The method of communication is RS-232, Bluetooth, and USB. The instrument and peripherals could be put into a follow-up bag and they have their own separate unit. When community doctor will go out for follow-up, they select the peripherals to light the weight of bag according to their needs.

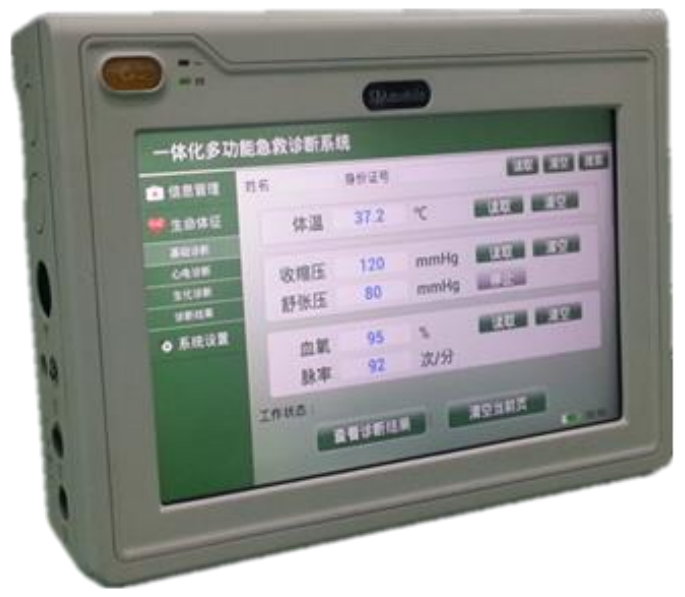

Figure 4. Integration Multifunction Health Management Terminal 


\section{Conclusion}

The paper presents an Intelligent Health Management Terminal which is based on Android system. It not only overcomes the shortages of terminal on market currently which has single function, low degree integration and non-portable, but also enhances the degree of intelligence The terminal adopts health information intelligent collection technology to achieve the detection of physiological indicators and biochemical indicators. The function of medical auxiliary diagnostic, remote export guide, electronic health document and health information management can help community doctor evaluate, screening and diagnose chronic for residents under the guidance of the expert doctors. The compact structure and portable design of terminal is taken to make sure that community doctor could go out for follow-up with it conveniently.

\section{Acknowledgement}

This work is supported by National Key Technology Research and Development Program of China (2015BAH09F01)

\section{References}

[1] Ye Ding, Shi Gang, Zhao Wei: The Design of the Intelligent Health Information Collection Terminal Which is Based on the Embedded Technology, Applied Mechanics and Materials, Vols. 433-435 pp 1448-1451, 2013

[2] Zhuang Keyu, Hu Jizhen, Zhang Chao: Data Acquisition Terminal Design of LV Customers Wireless Meter Reading System, hardware Technique, 2009.9, p23-26.

[3] Li Xueyi, Yan Zhuangzhi, Lv Haijiao: The Design of Smart Data Collection System for HER, Chinese Journal of Medical Instrumentation, Vol.33 No.6, 2009..

[4] Zhang Xiyang: The System of Mobile Telemedicine Consists of Mobile Unit, National University of Defense Technol, Changsha Hunan, China, 2005.

[5] Tian Lingling, Li Peng, Yu Fei: Intelligent Data Acquisition System Based on Embedded System, Journal of Qingdao University of Science and Technology, Vol.25 No.6 2004(12), p535-538.

[6] Wei Ning, Wang Yuhuan, Shi Yonghong: The design of data collection system which is based on GPRS wireless, Journal of Beijing Electronic Science and Technology Institute, Vol.14 No.4 2006(12) p.79-82.

[7] Karpov A.V., Frolov V.A.: Electronic Documentation of Present-Day Public Health ,KORUS, 2004 p.345-346.

[8] Yu Renshi, Hou Dacheng and Li Jing: Mater: the Design of Portable Medical Monitor Data Collection Terminal, Electronic Science and Technology, 2007(10), China. 